Archives

\title{
La compétence criminelle du présidial de Nantes sous le règne de Louis XIV
}

\section{Guy Saupin}

\section{(2) OpenEdition}

Journals

\section{Édition électronique}

URL : http://journals.openedition.org/ccrh/2172

DOI : $10.4000 /$ ccrh. 2172

ISSN : $1760-7906$

Éditeur

Centre de recherches historiques - EHESS

\section{Édition imprimée}

Date de publication : 20 octobre 1999

ISSN : 0990-9141

Référence électronique

Guy Saupin, "La compétence criminelle du présidial de Nantes sous le règne de Louis XIV », Les Cahiers du Centre de Recherches Historiques [En ligne], 23 | 1999, mis en ligne le 17 janvier 2009,

consulté le 10 décembre 2020. URL : http://journals.openedition.org/ccrh/2172 ; DOI : https://doi.org/ $10.4000 /$ ccrh. 2172

Ce document a été généré automatiquement le 10 décembre 2020.

Article L.111-1 du Code de la propriété intellectuelle. 


\title{
La compétence criminelle $d u$ présidial de Nantes sous le règne de Louis XIV
}

\author{
Guy Saupin
}

1 L'édit de création des présidiaux de janvier 1551, donné officiellement par Henri II pour désengorger les parlements, mais certainement aussi pour soutenir le trésor royal grâce à la vénalité des offices, se préoccupe beaucoup plus de préciser les conditions d'exercice de la justice civile que de fixer le détail de la justice criminelle. Daniel Jousse, conseiller au présidial d'Orléans, dans son célèbre Traité de la juridiction des présidiaux ${ }^{1}$, consacre également toute sa compétence à clarifier les principes de la procédure civile, en passant beaucoup plus vite sur la procédure criminelle.

2 Si cette étude se fixe comme but de présenter la compétence criminelle de la sénéchaussée présidiale de Nantes sous le règne de Louis XIV, ce n'est pas tant pour l'ambition systématique d'originalité que pour saisir une opportunité de clarifier cette question, sur la base d'une comparaison entre législation et pratique, grâce à un fonds exceptionnel d'archives conservé à la bibliothèque municipale de Nantes $^{2}$ et récemment analysé avec toute la finesse nécessaire par Joël Hautebert, spécialiste de l'histoire du droit ${ }^{3}$.

3 Le procureur du roi, André Boussineau, a laissé l'ensemble de ses conclusions définitives, tant civiles que criminelles, rendues devant le siège pendant toute la durée de sa charge (1678-1722). Cette source, avec ses 807 conclusions en matière criminelle, offre le précieux avantage de permettre une approche statistique fiable tant pour la typologie des délits que pour la ventilation des cas royaux, présidiaux et prévôtaux, perspective tout à fait aléatoire à partir des dossiers conservés dans la classique série B. Ces trois gros livres manuscrits sont complétés par un quatrième où le procureur du roi a rassemblé toutes les lettres rédigées entre 1679 et 1691, dans le cadre de son activité professionnelle. Celles-ci sont principalement adressées au procureur général du parlement de Bretagne dont la surveillance sur le déroulement procédurier au présidial de Nantes se trouve ainsi bien éclairée. 
4 Afin de caractériser les principes appliqués dans la qualification de la compétence criminelle dans un siège qui fait cohabiter deux cours regroupées dans une même compagnie, tout en étant en concurrence possible avec la juridiction de la maréchaussée, il convient de préciser les équilibres établis entre les deux cours siamoises, comme avec la prévôté des maréchaux, les justices subalternes et le parlement de Bretagne.

\section{Domination de la sénéchaussée et expansion de la présidialité}

La déclaration royale du 29 mai 1702 rappelle la distinction à opérer entre le civil et le criminel dans l'étendue géographique du ressort car pour la seconde compétence un présidial ne couvre que le ressort de la sénéchaussée principale à laquelle il est associé, laissant échapper les sénéchaussées secondaires dont les appels partent directement au parlement. Le siège de Nantes ne peut prétendre avoir autorité au criminel sur la sénéchaussée de Guérande, alors que celle-ci est parfaitement établie au civil ${ }^{4}$.

6 Selon les archives laissées par le procureur du roi Boussineau, les procédures relevant de la compétence traditionnelle de la sénéchaussée représentent les trois quarts des affaires traitées par le siège de Nantes. Un tel rapport provient de l'organisation juridique, avec ses privilèges, mais aussi des préférences affichées des justiciables. En Bretagne, les ecclésiastiques ne peuvent être jugés que par des officiers royaux et les officialités ne peuvent fonctionner sans la présence du juge criminel d'une juridiction royale. En suivant le principe breton du lien automatique entre fief et justice, les juges royaux ont compétence en première instance sur les habitants du fief du roi (six maisons dans Nantes) et sur les domiciliés des domaines des seigneuries tenues immédiatement en fief du roi, c'est-à-dire vassaux nobles et leurs fermiers ou métayers. Cependant, le poids de la sénéchaussée vient d'ailleurs. Les archives montrent cette cour en compétition avec des juridictions seigneuriales sur des procès qui ne sont pas des cas royaux. Cela s'explique par l'exercice du droit de prévention, mais aussi par la préférence de certains requérants pour la justice royale.

7 La sénéchaussée se réserve les cas royaux. Malgré l'effort de précision de l'ordonnance criminelle de 1670, il demeure toujours une petite marge d'interprétation rendant compte de légères variations dans le classement. Normalement, cela inclut les troubles du service divin, les hérésies, les émotions et séditions, le trafic de fausse monnaie, les malversations d'officiers, les enlèvements de personnes par force et les incendies volontaires visant à susciter du trouble public. La cour conserve aussi le privilège d'entérinement des lettres de rémission ${ }^{5}$ déjà précisé dans l'édit de Châteaubriant de 1554. Selon les ordonnances, la sénéchaussée peut juger en appel de la prévôté ou des juridictions seigneuriales à condition qu'il n'y ait pas de peine afflictive ${ }^{6}$. Cette pratique reste fort marginale car les justiciables préfèrent se pourvoir directement au parlement comme l'observe l'intendant en novembre $1717^{7}$. Les exemples de sentences contestées livrés par les manuscrits Boussineau illustrent des peines pécuniaires peu élevées ${ }^{8}$. Si la condamnation est le plus souvent confirmée, il arrive qu'elle soit diminuée ou même annulée.

8 L'édit de création de janvier 1551, en renvoyant aux précédentes ordonnances pour la définition de la présidialité criminelle, manque singulièrement de précision. Celle-ci n'est obtenue qu'avec l'édit de Moulins de février 1566 qui retient la même compétence 
criminelle que celle des prévôts des maréchaux. Le présidial étant juge de sa propre compétence, il se réfère à la définition des cas présidiaux reprise par l'ordonnance de 1670 avant d'être amputée par la déclaration royale de février 1731. Sont cités les crimes des vagabonds et des gens de guerre, les vols sur les grands chemins ou par effraction, les ports d'armes avec violence publique et séditions, les assassinats prémédités, les sacrilèges et les affaires de fausse-monnaie... Les cas présidiaux restent par la nature du crime des cas royaux dont ils ne se différencient parfois que par la qualité et le nombre des personnes, ainsi que par les moyens employés, ce qui laisse une légère liberté d'interprétation de la terminologie officielle. De plus, l'analyse des procédures souligne la propension du présidial à déborder de son champ réglementé.

9 Les gentilshommes, les secrétaires du roi et certains grands officiers comme les présidents présidiaux jouissent du privilège de requérir la grand'chambre du parlement. Toutefois, des nobles sont jugés à Nantes en dernier ressort pour des affaires d'enlèvement forcé et de désordre militaire tandis que d'autres le sont à charge d'appel pour le même type de délits.

Le relevé des conclusions définitives du procureur du roi Boussineau permettent une évaluation statistique de l'importance des cas présidiaux ${ }^{9}$. Sur 807 conclusions rendues de 1679 à 1722,169 s'inscrivent dans la présidialité, soit $21 \%$. En additionnant 47 jugements prévôtaux, on arrive à $27 \%$ de procès jugés en dernier ressort, c'est-à-dire respectivement quatre ou cinq par an en moyenne. Pour 233 procès relevés sur l'ensemble du XVII ${ }^{\mathrm{e}}$ siècle, la compétence, de par la qualité des délinquants, est établie à 102 reprises $(43,8 \%)$. Les affaires concernent en priorité des vagabonds (75), peut-être parce que les magistrats ont une définition laxiste du vagabondage, incluant même la complicité. Les errants sanctionnés sont plutôt des hommes jeunes, originaires des provinces de l'Ouest. Les récidivistes, déjà condamnés par une institution statuant en dernier ressort, relèvent aussi du présidial. Si des enquêtes sont menées dans les greffes criminels, la preuve est surtout recherchée dans l'examen des traces corporelles des peines déjà infligées. Les récidivistes condamnés par le présidial ou le parlement et ayant enfreint leur ban sont jugés présidialement, sauf si la cour de Rennes fait valoir son droit de continuité. Les crimes des gens de guerre ne peuvent être que ceux des troupes de passage, à l'exception des soldats en garnison permanente qui bénéficient toujours du droit d'appel, et uniquement pour des crimes de soldat à habitant et non pas de soldat à soldat. Si la procédure en dernier ressort est appliquée pour des officiers inférieurs nobles, elle n'est jamais utilisée pour la petite noblesse du ban et de l'arrière-ban.

11 Malgré la prétention à la précision de l'ordonnance criminelle de 1670, l'interprétation des termes laisse une certaine marge de manœuvre à l'expansionnisme du présidial. Pour 143 procédures où la compétence est justifiée par la nature du crime (60,4 \% du corpus), les vols de toute nature (deux tiers) sont suivis de loin par les assassinats prémédités et les affaires de fausse-monnaie. Ces proportions ne sont que des indicateurs de tendance car la ventilation par cause n'est pas chose aisée puisque le délit additionne souvent plusieurs infractions ${ }^{10}$. Les juges nantais utilisent volontiers la préméditation pour inclure les infanticides, ce qui est contraire aux ordonnances. La même propension s'observe à propos des vols de biens profanes dans les églises considérés comme sacrilèges. Pour qu'une assemblée illicite - cas royal - soit saisie comme cas présidial, il faut qu'il y ait port d'armes, mais les juges nantais ne s'embarrassent guère de cette distinction, dans une approche très floue de la notion d'assemblée illicite qui couvre tout attroupement de vagabonds ou de soldats. Le fait d'avoir trois ou quatre accusés 
transforme ainsi le rapt de violence de simple cas royal en cas présidial, de même que tout vol effectué en groupe, même sans port d'armes.

La compétence du présidial dépend aussi de la pluralité des crimes et des délinquants. Si

l'infraction est qualifiée pour sa nature, tous les accusés sont jugés présidialement, sauf s'il se trouve une personne qui ne peut être jugée en dernier ressort. Le privilège de cet accusé couvre alors tous ses complices et le jugement ne peut être rendu qu'à charge de l'appel. Inversement, si le délit est qualifié en vertu de la qualité des délinquants, les juges nantais ont comme principe de faire prévaloir le statut de l'accusé relevant de la présidialité. Un errant, un récidiviste ou un militaire en déplacement entraînent ainsi tous leurs complices dans ce type de procédure. De même, le présidial peut dessaisir un juge inférieur pour juger conjointement dans la procédure de dernier ressort tous contrevenants ayant commis plusieurs délits dont un seul est présidial. Ce jugement de compétence s'effectuant au début de la procédure, un individu peut se trouver condamné présidialement pour un crime qui ne relève pas de cette catégorie, même s'il s'avère finalement impossible de prouver sa culpabilité dans l'affaire qui lui a valu d'être rangé dans cette procédure.

La localisation géographique des infractions jugées en dernier ressort témoigne d'une sur-représentation de l'espace urbain puisque 48 délits sur 110 cas analysables se situent dans la ville et ses faubourgs. La faiblesse des forces de police et des rémunérations accordées aux sergents royaux expliquent la décroissance très rapide de leur efficacité. Les 62 délits en espace rural, dont 14 sur les grands chemins partant de Nantes, sont répartis exactement par moitié au nord et au sud de la Loire.

\section{Mise sous tutelle de la juridiction de la maréchaussée}

14 Si la compétence criminelle de la juridiction du prévôt des maréchaux établie en Bretagne par l'édit de 1533 sert de référence au présidial, cette cour ne peut cependant rendre la justice qu'avec la participation de juges d'une autre juridiction, après que sa compétence ait été reconnue par le présidial ${ }^{11}$.

15 Jusqu'en 1708, la maréchaussée est présente dans le comté nantais sous la forme d'une brigade composée d'une dizaine d'archers et d'un greffier, placée sous l'autorité d'un lieutenant du prévôt, ce dernier restant basé à Rennes. Cet officier a pour mission de saisir des criminels et de procéder aux jugements prévôtaux. Théoriquement, un assesseur, un procureur du roi et un greffier doivent le soutenir dans cette dernière tâche. Si un édit de décembre 1594 a bien créé une charge d'assesseur, celle-ci n'a jamais été pourvue à Nantes avant sa transformation en commission en 1720. La fonction est assurée en réalité par un magistrat du présidial, dans l'ordre hiérarchique, concrètement essentiellement par le juge magistrat criminel. Le procureur du roi au présidial sert également à la prévôté.

Justice d'exception, la maréchaussée assure avant tout des missions de police au service des juridictions ordinaires, mais le procureur du roi Boussineau n'est guère satisfait des services rendus par le lieutenant, Vivier du Plessis, à la fin du xvii siècle. Elle ne juge qu'en dernier ressort des cas identiques aux cas présidiaux, mais elle a aussi compétence sur le ressort de la sénéchaussée de Guérande, à la différence du présidial. Ceci reste assez théorique puisque la brigade répugne en fait à trop s'éloigner de Nantes. Si la répartition des lieux d'infraction des 34 cas prévôtaux recensés est sensiblement la même que celle 
des cas présidiaux déjà évoquée en ce qui concerne le rapport ville-campagne ( $44 \%$ - $56 \%$ ), elle suggère une attention beaucoup plus poussée sur le nord de la Loire, sans qu'il soit possible de proposer une interprétation. La convergence entre les données extraites des liasses de procédures criminelles (133 procès entre 1603 et 1679 donnent $71 \%$ de cas présidiaux) et les conclusions de Boussineau (216 procès de 1680 à 1722 donnent $78 \%$ de cas présidiaux), permet d'établir une proportion de trois procès présidiaux pour un procès prévôtal dans le comté nantais.

17 La maréchaussée ne peut être compétente qu'à condition d'avoir, de sa propre initiative, arrêté elle-même le délinquant. Le prisonnier étant conduit aux prisons royales du Bouffay, le présidial doit alors se prononcer sur la compétence du prévôt, dans un délai de trois jours selon l'ordonnance de 1670, ce qui rend plus exceptionnelles, sans les supprimer totalement, les attentes démesurées avant jugement. Un long moment peut cependant s'écouler entre le jugement de compétence et l'engagement réel de la procédure. Des plaintes semblent récurrentes du début du XVII ${ }^{\mathrm{e}}$ siècle au début du XVIII ${ }^{\mathrm{e}}$ siècle et l'intendant Feydeau de Brou n'établit pas de différence entre les deux partenaires dans son rappel à l'ordre de 1722 .

L'examen de la compétence se fait à l'interrogatoire, le prévenu pouvant argumenter une récusation de la prévôté. Les archives nantaises ne livrent toutefois aucun exemple d'abandon d'une procédure prévôtale, ce qui s'explique par la tutelle ultérieure exercée en réalité par le présidial qui ne prend ainsi aucun risque en se montrant coopératif. Cette décision est un jugement présidial en dernier ressort qui nécessite la présence de sept juges avec généralement le lieutenant général, le juge criminel et des conseillers, le lieutenant du prévôt n'ayant ici pas voix délibérative.

Selon la législation royale, les étapes de l'instruction devraient être conduites par l'assesseur, en collaboration avec le lieutenant de la prévôté. À Nantes, cette charge n'ayant jamais été pourvue avant 1720 , le travail en revient au juge criminel ou en son absence au lieutenant particulier civil et criminel ou encore à un conseiller. Selon la formulation des actes, les interrogatoires, récolements ou confrontations, comme la question préalable se déroulent devant le lieutenant de la prévôté et en présence du juge criminel ou de son remplaçant avant 1670, devant le rapporteur et en présence des précédents après 1670 .

Tout jugement prévôtal requérant la présence d'au moins sept juges, le lieutenant du prévôt a-t-il voix délibérative et est-il compté parmi cet ensemble? Le dernier interrogatoire d'un jugement en dernier ressort doit être mené devant l'ensemble des magistrats devant rendre le jugement. Le rapport et les conclusions définitives du procureur du roi y sont présentés. La sentence est formulée le jour même. À Nantes, tous les actes antérieurs à 1678 montrent que le lieutenant du prévôt ne participe pas à cette ultime séance présidée par le président du présidial ou le lieutenant général. La décision est rendue par «les gens tenant le siège présidial » comme une «sentence et jugement présidial en dernier ressort suivant les édits du roi ", avec toutefois introduction de la mention «dit et disons que le lieutenant du prévôt doit par son jugement prévôtal déclarer (N...) atteint et convaincu... » immédiatement avant l'énoncé de la peine. Ainsi une seconde sentence, rendue dans un second temps par le seul lieutenant, reprend exactement les mêmes termes.

21 L'ordonnance criminelle de 1670 parvient à faire évoluer la pratique dans le sens d'une association intégrale au bout d'une dizaine d'années. Une première sentence datant de 
1685 est signée par le lieutenant qui s'ajoute aux sept juges du présidial, comme voix supplémentaire. Accepté dorénavant au dernier interrogatoire, il ne peut cependant prétendre le présider, cet honneur restant le privilège du président du présidial ou du lieutenant général. Dans un conflit en 1734 avec le chancelier d'Aguesseau, les magistrats nantais justifient cet ancien usage par l'insuffisance des compétences juridiques d'un officier non gradué en droit $^{12}$. Ce rééquilibrage interne favorisant une meilleure reconnaissance des officiers prévôtaux au XVIII ${ }^{\mathrm{e}}$ siècle ne doit pas masquer l'essentiel : le maintien de l'illusion d'une procédure prévôtale qui demeure sévèrement contrôlée en réalité par le siège présidial.

\section{Relations avec les autres juridictions}

Depuis l'édit de Châteaubriant d'octobre 1565 qui a supprimé les trois petites barres royales du Gavre, de Loyaux (Fresnay) et Touffou (Le Bignon), il ne demeure plus que la prévôté de Nantes comme juridiction royale dans le ressort du présidial en matière criminelle. Très peu de terres en relevant en proche fief, l'essentiel de la compétence s'exerce sur les tribunaux seigneuriaux dont on sait la forte densité en Bretagne. Selon André Giffard, il faut compter 592 justices dont 66 ecclésiastiques et 526 laïques réparties sur 221 paroisses dans l'évêché de Nantes ${ }^{13}$. Le droit de juridiction étant attaché au fief, les limites des justices seigneuriales très nombreuses évoluent selon la transmission des fiefs. Chargé par le procureur du parlement de dresser une liste des hauts justiciers du ressort du présidial, le procureur du roi Boussineau avoue ne pas connaître « les officiers qui y exercent..., non plus que les bourgs et villes qui en dépendent $»^{14}$. Bien que les juges seigneuriaux soient compétents en qualité de juges des lieux, sauf pour ce qui touche aux cas royaux et présidiaux ou à la qualité des personnes comme les ecclésiastiques et les commis aux devoirs, bien des affaires arrivent cependant directement au présidial par choix des justiciables, surtout lorsqu'il existe des liens de parenté entre les officiers seigneuriaux et les accusés ou lorsque ces derniers sont fermiers de la seigneurie.

D'autres sont saisies au nom du droit de prévention, sous réserve que le seigneur ne fasse pas valoir les droits de son tribunal qui sont liés à sa rapidité de réaction après la date du délit. Le délai de quinze jours fixé par la coutume de Bretagne de 1580 est toutefois ramené à trois jours par l'ordonnance de 1670. Le droit de reporter tous les frais de procédure sur la justice seigneuriale existe en Bretagne pendant tout le XVII ${ }^{\mathrm{e}}$ siècle, même après la contraction sévère du temps de réaction en 1670. À partir de cette ordonnance, les juges seigneuriaux et royaux inférieurs, dont les appels partent directement au parlement, doivent envoyer deux fois par an un extrait de leur registre criminel. Les plaintes de Boussineau montrent que cette opération, même devenue annuelle, ne s'opère pas aisément ${ }^{15}$. Son insistance s'explique surtout par la volonté de mieux connaître les récidivistes.

La prévôté, très ancienne juridiction ducale devenue royale, dont le juge unique porte le titre de conseiller du roi, juge prévôt, magistrat ordinaire civil et criminel, conservateur des privilèges de l'université, lieutenant général de police de Nantes (1699), a des relations beaucoup plus tendues avec le présidial. En qualité de juge des lieux sur les paroisses de son ressort et sur les terres relevant du domaine royal dans l'enceinte de la ville et faubourgs, sa compétence criminelle est en tout point égale à celle des seigneurs hauts justiciers. Cette conflictualité plus grande résulte certainement de la concentration sur l'espace urbain ${ }^{16}$. Selon Gérard Mellier, subdélégué de l'intendant depuis 1710 et 
maire de Nantes de 1720 à 1729, Charles IX, par l'ordonnance de Moulins de 1566, avait supprimé la prévôté «à la sollicitation des officiers du présidial de Nantes » et ne l'avait rétablie en 1568 qu'après un plaidoyer pour une charge « établie depuis plus de 400 ans pour connaître principalement des devoirs de prévosté..., et régler la police de la ville $»^{17}$. Les justifications mises en avant prouvent le maintien d'une hostilité de principe envers une concurrence jugée inutile en termes de justice criminelle. Par ailleurs, sa position de juge unique oblige le prévôt à solliciter l'aide des magistrats du présidial pour tout procès ouvrant sur des peines afflictives et infamantes. Normalement, le lieutenant civil et criminel s'y déplace, un avocat étant constitué comme troisième juge. Il y préside la séance dans laquelle le prévôt n'est que le rapporteur. La désinvolture avec laquelle les magistrats du siège traitent l'officier royal ne fait qu'envenimer une tension qui ne disparaît qu'avec la suppression de la prévôté en 1749.

Dans les délits communs (injures verbales, bagarres et disputes de dîmes) dont la sanction est limitée à une réparation pécuniaire, le présidial et l'officialité sont en concurrence. Le renvoi exigé par l'official doit lui être consenti, sauf si l'affaire est particulièrement grave. Les cas privilégiés, qualifiés par la possibilité de peines afflictives, sont par contre jugés conjointement par le juge criminel et l'official, ce qui évite des querelles de compétence ${ }^{18}$. L'inégalité réside dans le fait que l'official saisi d'un cas privilégié doit avertir obligatoirement le juge criminel alors que la réciproque n'est pas vraie: le présidial n'ayant pas reçu de demande de renvoi de l'officialité est compétent pour juger seul. Si la procédure est conjointe, chaque juridiction rend une sentence particulière selon ses critères d'appréciation.

Dans les relations avec le parlement de Bretagne ${ }^{19}$, il convient de séparer les procédures relevant de la compétence classique de la sénéchaussée et celles qui sont liées aux cas présidiaux et prévôtaux.

En ce qui relève de l'activité de la sénéchaussée, le parlement renvoie à la cour un certain nombre d'affaires dont elle a été saisie par méfiance des plaignants envers les juges seigneuriaux ou par méconnaissance des véritables ressorts des nombreuses cours. Lorsque le ministère public est seul partie, le parlement accorde une commission au juge criminel pour procéder au jugement à charge de l'appel, procédure également utilisée pour des procès jugés à la Tournelle et demandant une information complémentaire sur Nantes auprès des victimes. Les appels au parlement s'appliquent aussi bien aux jugements en cours d'instruction qu'aux sentences définitives ${ }^{20}$. Ils sont formés aussi bien par les accusés, pour les premiers, que par la partie publique ou la partie civile pour les seconds.

29 Toutes les condamnations à peine afflictive (peine de mort, la question avec ou sans réserve de preuves, les galères perpétuelles et à temps, le bannissement perpétuel et à temps, le fouet, le carcan, le pilori et l'amende honorable) sont rendues avec appel obligatoire au parlement, exercé par le procureur du roi, surtout s'il estime que la sanction reste insuffisante. Pour les autres jugements, l'initiative en revient au condamné dans l'espoir d'une atténuation ou à la partie civile pour un alourdissement.

En principe, un parlement ne peut aller contre les jugements présidiaux en dernier ressort dont la cassation ne relève que du Grand Conseil. Cependant, le privilège de juger en dernier ressort ne remet pas en cause le principe de la suprématie de la jurisprudence et de la prérogative réglementaire de la cour souveraine. Les archives nantaises montrent ainsi que le parlement de Bretagne parvient à contourner légalement une impossibilité 
d'intervention. Le procureur général de Rennes surveille de très près toutes les questions de compétence pour lesquelles il est informé par l'envoi de la liste des procédures tous les six mois depuis 1670, les plaintes des cours inférieures s'estimant lésées et la correspondance suivie avec le procureur du roi à Nantes dont les papiers Boussineau donnent une illustration saisissante ${ }^{21}$. Le parlement peut surtout agir sur les actes de procédure antérieurs au jugement de compétence, comme le décret de prise de corps, la permission d'informer ou l'arrestation abusive. De même, une prise à partie pour malversation est toujours possible contre les juges présidiaux et le procureur du roi tout au long de la procédure. Boussineau évoque ce problème dans une affaire de vol de bestiaux en 1689, ce qui embarrasse messieurs du présidial car le délit n'est pas reconnu officiellement comme un cas présidial, même si la jurisprudence nantaise tend à l'assimiler'22.

31 Au terme de cette étude sur le travail effectué par le présidial de Nantes en matière de justice criminelle sous le règne de Louis XIV, quelques caractéristiques essentielles méritent d'être soulignées.

Les conclusions définitives recensées par le procureur du roi montrent en moyenne 19 affaires traitées par an, dont quatorze au nom d'une compétence classique de sénéchausssée et cinq pour une présidialité incluant un cas prévôtal. Si le poids relatif de la juridiction royale ancienne demeure prépondérant, notons la tendance très nette à favoriser, autant que faire se peut, les cas de présidialité, sans doute pour conforter ce privilège de juger en dernier ressort ${ }^{23}$. Dans le comté nantais, la procédure prévôtale n'est qu'une illusion puisque elle demeure entièrement contrôlée par le siège présidial jusqu'à un timide rééquilibrage à partir de 1720 . Comme tribunal intermédiaire, le présidial joue effectivement son rôle de grignotage de la puissance seigneuriale, non seulement en profitant du resserrement de la réglementation monarchique, mais aussi de la préférence affichée des justiciables dans certains procès où les agents seigneuriaux sont en cause. La surveillance parlementaire de la régularité des procédures n'a jamais occasionné de conflit d'importance.

Le présidial de Nantes intéresse particulièrement les habitants de la ville et faubourgs, ce qui reflète un décalage saisissant entre l'emprise judiciaire royale dans les mondes urbains et ruraux, d'autant plus que l'impact dans les campagnes décroît très rapidement lorsqu'on s'éloigne de la ville. La phase observée s'inscrit nettement dans la période d'influence forte du présidial, avant les problèmes de chute d'activité et de détérioration d'image qui surviennent ensuite dans le cours $d u x_{\text {xiII }}$ siècle. Le prestige social des officiers demeure encore considérable. La compagnie est toujours placée sous l'influence des Charette, famille qui domine la vie politique nantaise au $\mathrm{XVII}^{\mathrm{e}}$ siècle $^{24}$, en monopolisant l'office de sénéchal depuis 1587 et en le cumulant avec celui de président du présidial depuis 1687, le tout jusqu'en 1740. 


\section{NOTES}

1. Daniel Jousse, Traité de la juridiction des présidiaux tant en matière civile que criminelle, Paris, 1757, $2^{\mathrm{e}}$ éd., Paris, 1764.

2. Bibliothèque municipale (BM) de Nantes, Papiers Boussineau. Trois volumes regroupent les conclusions rendues par ce procureur du roi. Le manuscrit 307 pour la période 1679-1700, le 308 pour 1702-1712, le 309 pour 1713-1722. Quelques lacunes pour 1700-1702. Un quatrième manuscrit (310) rassemble les lettres expédiées au sujet de problèmes rencontrés dans sa fonction.

3. Joël Hautebert, La Jurisprudence criminelle de la sénéchaussée présidiale de Nantes, 1551-1750, 2 vol., thèse de droit, Rennes I, 1996.

4. René Giffard, Essai sur les présidiaux bretons, Paris, 1902, 112 p., p. 12.

5. Dans les conclusions définitives de Boussineau: 56 cas et 39 juridictions citées dont le présidial huit fois, les régaires six, la prévôté quatre, les juridictions de Donges et Machecoul trois, le marquisat de Goulaine deux et toutes les autres une seule fois.

6. La juridiction de première instance est connue dans 34 cas : la prévôté apparaît huit fois, le marquisat de La Guerche six, le marquisat de Goulaine trois, les juridictions de Bougon en Bougunenais, de Vay et de la vicomté de Donges deux et les autres une fois.

7. Archives départementales d'Ile-et-Vilaine (ADIV), C 1818, lettre du 22 novembre 1717.

8. Joël Hautebert, op. cit., p. 58.

9. Ibid., p. 62-63.

10. Ibid., p. 70.

11. Eliane Bertin-Mourot, La Maréchaussée en Bretagne au XVIII ${ }^{\mathrm{e}}$ siècle, 1720-1789, thèse de droit, t. I, Rennes I, 1969, p. 22.

12. Archives départementales de Loire-Atlantique (ADLA), B 6751, lettre du 10 février 1734.

13. André Giffard, Les Justices seigneuriales en Bretagne au XVIII ${ }^{\mathrm{e}}$ siècle, Paris, 1902, 376 p., p. 307.

14. BM Nantes, manuscrit 310, lettre du 27 novembre 1689, p. 589.

15. BM Nantes, manuscrit 310, lettre du 10 février 1686 , p. 219 ; manuscrit 169 , lettre du $1^{\text {er }}$ septembre 1685.

16. Archives municipales de Nantes, FF 3, réponse ( 25 novembre 1717) du greffier de la prévôté au mandement de Mellier. «La prévôté de Nantes est un siège royal dont la juridiction s'étend dans toute l'enceinte de ladite ville qui comprend sept paroisses, en ce qui relève du proche fief du roi, lequel fief est enclavé avec ceux de plusieurs seigneurs, du chapitre, de la commanderie de Saint-Jean et de Sainte-Catherine, du prieuré de Toutes-Joies, de Sainte-Julite et de Boisgarant, de la Madeleine-en-Bois, et qui ont leurs juridictions, ce qui contient près de la moitié de la ville ».

17. Gérard Mellier, Essai sur l'histoire de la ville et du comté de Nantes, manuscrit de 1719, publié par Léon Maître, Nantes, 1872, 145 p., p. 134.

18. BM Nantes, manuscrit 307, p. 7 ; m. 309, p. $54 ;$ m. 310, p. 94.

19. Marie-Yvonne Crépin, Le Contrôle du parlement de Bretagne sur l'administration de la justice, thèse droit, Rennes I, 1979.

20. Ibid., p. 106-107. La plupart des prises à partie sont fondées sur l'irrégularité des procédures, telles l'incompétence et l'arrestation injustifiée du requérant. Elles sont fréquentes, ce qui ne veut pas dire qu'elles soient facilement admises.

21. BM Nantes, manuscrit 310, p. 20, lettre du 24 juin 1679.

22. Ibid., p. 525, lettre du 22 février 1689. 
23. Sylvain Soleil, Le Siège royal de la sénéchaussée et du présidial d'Angers, Rennes, 1997, 383 p., p. 114-126. À titre de comparaison, dans une province voisine, dans la périphérie du ressort du parlement de Paris.

24. Guy Saupin, Nantes au XVII ${ }^{\mathrm{e}}$ siècle. Vie politique et société urbaine, 1598-1720, Rennes, 1996, $244 \mathrm{p}$. 